\title{
Elemente der Planeten (305) und (306).
}

Planot (305) (Charlois Fobr. 16).

Aus den Beobachtungen Febr. 16 Nizza, Febr. 28 Hamburg und Mărz 19 Nizza ergeben sich die Elemente:

Epoche 189 I März 19.5 M. Z. Berlin

$$
\begin{aligned}
& M=34^{\circ} 4^{\circ} 55^{\circ} \circ \\
& \omega=254 \quad 2 \quad 21.6 \\
& \delta=21034 \quad 16.9 \\
& i=42620.2 \\
& \varphi=\begin{array}{lll}
\text { I } & 6 & 39.4
\end{array} \\
& \mu=650.924 \\
& \log a=0.490984
\end{aligned}
$$

Planet (306) Onitas.

Aus den Beobachtungen März I Rom, März 16 und 28 Wien, und April 9 (Paris, Klumpke) folgen die Elemente :

Epoche 1891 April 9.5 M. Z. Berlin.

$$
\begin{aligned}
& M=235^{\circ} 2 \mathrm{I}^{\circ} \quad 4^{\prime \prime} 7 \\
& \omega=164 \text { I5 } 8.4 \\
& \delta=14132 \quad 37.2 \\
& i=71420.0 \\
& \varphi=84239.6 \\
& \mu=977^{\prime 2685} \\
& \log a=0.3733^{285}
\end{aligned}
$$

Mittlere Oerter : März $16 \mathrm{~d} \lambda=+1: 3 \mathrm{~d} \beta=-2: \mathrm{I}$

$$
\begin{array}{lll}
(B-R) & 28 & -0.7
\end{array}
$$

Der Planet (306) Unitas wird in den Oppositionen, die nahe mit dem Perihel zusammenfallen, recht hell, etwa 9. Grösse, werden.

Berlin 1891 April 23.

A. Berberich.

\section{Vermischte Nachrichten.}

(Auszug aus dem Anzeiger der Wiener Akademie 189r Nr. I und V.)

R. König. Bestimmung der Bahndes Cometen 1857 III. Der an 22. Juni 1857 von Klinkerfues zu Göttingen aufgefundene teleskopische Comet konnte nur während eines Zeitraums von 28 Tagen beobachtet werden, da er am r8. Juli bereits in das Perihel kam und zu dieser Zeit bei heller Dämmerung unterging. Auf Grund aller zu Gebote stehenden Beobachtungen und unter Neurechnung der Sonnenörter und der Reductionselemente, sowie nach sorgfaltiger Bestimmung der Positionen der benutzten Vergleichsterne wurde als wahrscheinlichste Bahn die folgende Parabel erhalten :

$$
\begin{aligned}
& T=1857 \text { Juli } 18.0114722 \text { M. Z. Berlin } \\
& \omega=134^{\circ} 3^{\prime} \times 7^{\prime \prime} \cdot 76 \\
& \delta=234132.16 \text { M. Aequ. } 1857.0 \\
& i=121 \quad 1 \quad 0.54 \\
& q=0.367535^{\circ}
\end{aligned}
$$

Eine Untersuchung, ob die Einführung einer Excentricitüt eine befriedigendere Darstellung der Beobachtungen als diese Parabel zulasse, führte zu dem Resultate, dass in der That eine schwache Andeutung einer elliptischen Bahn vorliege, dass jedoch einigermaassen sichere Angaben hierüber nicht gemacht werden können.
S. Oppenheim. Bestimmung der Bahn des Planeten (290) Bruna.

Die Elemente dieses von Herrn Dr. J. Palisa, Adjuncten der k. k. Universităts - Sternwarte, am 20. März 1890 entdeckten und bis zum I I. Mai beobachteten Planeten sind:

(290) Bruna.

$$
\begin{aligned}
& \text { Epoche } 1890 \text { Mai 7.5 M. Z. Berlin. } \\
& L=170^{\circ} 40^{\circ} 37^{\prime \prime} 3 \\
& M=564922.1 \\
& \pi=113515.2 \\
& 8=101841.2 \\
& i=22 \times 328.3 \\
& \varphi=15422.7 \\
& \log a=0.368066 \\
& \mu=995^{\prime \prime} 1925
\end{aligned}
$$

Anzeige betr. ein nenes General-Register zu den Astr. Tachr. Ein General-Register der Astronomischen Nachrichten zu Band 81 bis 120, zusammengestellt von den Herren Dr. H. Kreutz und Dr. R. Schorr ist kürzlich erschienen. Der Preis ist auf 25 Mark festgestellt. Bestellungen nimmt die Expedition der Astronomischen Nachrichten in Kiel sowie die Buchhandlung von W. Mauke Söhne in Hamburg entgegen. A. Krueger.

Inhalt zu Nr. 3036. E. Becker. Ueber einige Versuche von Durchgangsbeobachtungen nach dem neuen Repsold'schen Verfahren. 185. $\boldsymbol{R}$. Luther. Kreismikrometer-Beobachtungen von kleinen Planeten am Siebenflisser 2n Dilsseldorf. 189. $-E$. Millosevich. Osservazioni del pianetino (303) Josephina. 193. - F. Tebbutt. Star-Occultations observed at Windsor N. S. Wales in 1890. 193. - F. Porro. Osservazioni di Stelle Variabili a lungo periodo. 195. - B. von Engelhardt: Beobachtungen des Cometen 1890 II. 197. - A. Krueger. Ephemeride des Wolf schen Cometen 1884 III fur die zweite Eracheinung 1891 ... 197. A. Berberich. Elemente der Planeten (305) und (306). 199. - Vermischte Nachrichten, 199. - Anzeige. I99. 